\title{
Cycling Exercise Training Enhances Mitochondrial Bioenergetics of Platelets in Patients with Peripheral Arterial Disease: A Randomized Controlled Trial
}

Ming-Lu Lin, $\mathrm{PhD}^{1}$; Tieh-Cheng Fu, MD, $\mathrm{PhD}^{2+}$; Chih-Chin Hsu, MD, $\mathrm{PhD}^{2}$; Shu-Chun Huang, MD, $\mathrm{PhD}^{3}$; Yu-Ting Lin, $\mathrm{PhD}^{1}$; Jong-Shyan Wang, $\mathrm{PhD}^{1,2,4 *}$

${ }^{1}$ Healthy Aging Research Center, Graduate Institute of Rehabilitation Science, Medical Collage, Chang Gung University, Tao-Yuan, Taiwan

${ }^{2}$ Department of Physical Medicine and Rehabilitation, Chang Gung Memorial Hospital, Keelung, Taiwan

${ }^{3}$ Department of Physical Medicine and Rehabilitation, Chang Gung Memorial Hospital, TaoYuan, Taiwan

${ }^{4}$ Research Center for Chinese Herbal Medicine, College of Human Ecology, Chang Gung University of Science and Technology, Tao-Yuan, Taiwan

${ }^{+}$Equal contribution as the first author

Short title: Exercise effect on platelet mitochondrial function in PAD patients

* Please send correspondence to:

Professor, Jong-Shyan Wang

Graduate Institute of Rehabilitation Science

Chang Gung University

259 Wen-Hwa $1^{\text {st }}$ Road, Kwei-Shan, Tao-Yuan, 333, Taiwan

Fax \# 886-3-2118700

E-mail address: s5492@mail.cgu.edu.tw 


\begin{abstract}
Exercise training influences the risk of vascular thrombosis in patients with peripheral arterial disease (PAD). Mitochondrial functionalities in platelets involve the cellular bioenergetics and thrombogenesis. This study aimed to elucidate the effect of cycling exercise training (CET) on platelet mitochondrial bioenergetics in PAD patients. Forty randomly selected patients with PAD engaged in general rehabilitation (GR) with CET (i.e., cycling exercise at ventilation threshold for $30 \mathrm{~min} /$ day, 3 days/week) (GR+CET, $\mathrm{n}=20$ ) or to a control group that only received GR course $(n=20)$ for 12 weeks. Systemic aerobic capacity and platelet mitochondrial bioenergetics that included oxidative phosphorylation (OXPHOS) and electron transport system (ETS) were measured using automatic gas analysis and high-resolution respirometry, respectively. The experimental results demonstrated that GR+CET for 12 weeks significantly (i) elevated $\mathrm{VO}_{2 \text { peak }}$ and lowered $\mathrm{V}_{\mathrm{E}}-\mathrm{VCO}_{2}$ slope, (ii) raised resting ankle-brachial index and enhanced cardiac output response to exercise, (iii) increased the distance in 6-minute walk test and raised the Short Form-36 physical/mental component scores, and (iv) enhanced capacities of mitochondrial OXPHOS and ETS in platelets by activating FADH2 (Complex II)dependent pathway. Moreover, changes in $\mathrm{VO}_{2 \text { peak }}$ levels were positively associated with changes in platelet OXPHOS and ETS capacities. However, no significant changes in systemic aerobic capacity, platelet mitochondrial bioenergetics, and health-related quality of life (HRQoL) occurred following GR alone. Hence, we conclude that CET effectively increases the capacities of platelet mitochondrial bioenergetics by enhancing Complex II activity in patients with PAD. Moreover, the exercise regimen also enhanced functional exercise capacity, consequently improving HRQoL in PAD patients.
\end{abstract}

Keywords: peripheral arterial disease, exercise, platelet, mitochondria 


\section{Introduction}

Peripheral arterial disease (PAD) is a manifestation of atherosclerosis or thrombosis that causes chronic narrowing of peripheral arteries, consequently reducing the capacity of blood flow to contracting muscles in legs [1]. Moreover, reduced exercise capacity negatively affects the ability of PAD patients to perform the activities required for daily life, further decreasing their independence and quality of life [2,3]. However, exercise training may not only improve physical performance but also reduce vascular thrombotic risk in patients with PAD [2,3].

Platelets play a critical role in thrombogenesis of PAD patients [4,5]. Platelet mitochondrial functionality are mainly involved in the cellular redox balance and activation, thereby modulating thrombogenesis [6-8]. Cycling exercise training (CET) improves aerobic fitness, concurrence with reducing the risk of major vascular thrombotic events in patients with circulatory disorders $[9,10]$. According to our early studies, moderateintensity exercise training on a bicycle ergometer depressed platelet adhesion/aggregation at rest and attenuated the enhancement of platelet reactivity caused by acute strenuous exercise $[11,12]$. Recently, our investigation further demonstrated that CET improved the capacity for platelet mitochondrial bioenergetics $[13,14]$ and reduced platelet-induced thrombin generation [13] in healthy sedentary people [13] or heart failure (HF) patients [14]. However, the effects of CET on platelet mitochondrial bioenergetics in PAD patients have not yet been established.

The electron transport complexes in mitochondrion are interconnected in mitochondrial inner membrane and turn into respiratory supercomplexes [15]. Mitochondrial structure and function are disrupted as isolating independent organelles from whole cells [16]. A highresolution respirometry can measure mitochondrial functionality by the addition of exogenous substrates and inhibitor to the permeablized cells [17]. Recently, we have established two novel reference protocols of the substrate-uncoupler-inhibitor titration (SUIT-RPs), those were employed to determine the capacities of mitochondrial oxidative phosphorylation (OXPHOS) and electron transport system (ETS) in platelets, respectively [18]. Therefore, the present study further evaluated how CET effects systemic aerobic capacity and mitochondrial OXPHOS and ETS activities of platelets in PAD patients.

To answer the abovementioned questions, this study clearly assessed how CET (i.e., cycling exercise at ventilation threshold for $30 \mathrm{~min} /$ day, 3 days/week for 12 weeks) affects systemic aerobic capacity and mitochondrial OXPHOS and ETS activities of platelets in patients with PAD. The present study aimed to establish an effective exercise regimen for improving aerobic capacity and to enhance platelet mitochondrial functionality in PAD patients. 


\section{Methods}

\subsection{Participants}

Fifty-two patients diagnosed with PAD have been surveyed for the interventions from April 1, 2018 to December 31, 2019 at the Chang Gung Memorial Hospital, Keelung, Taiwan. Inclusion criteria in this investigation were listed as follows: (i) $>20$ years old, (ii) PAD for more than 2 weeks; (iii) ankle-brachial index $(\mathrm{ABI}) \leq 0.9$, and (iii) active voluntary exercise. Exclusion criteria were listed as follows: (i) less than 20 years old; (ii) unstable angina; (iiii) systolic blood pressure (SBP) at rest is greater than $200 \mathrm{~mm} \mathrm{Hg}$ or diastolic blood pressure (DBP) greater than $110 \mathrm{~mm} \mathrm{Hg}$; (iv) the orthostatic blood pressure drop is greater than $20 \mathrm{~mm}$ $\mathrm{Hg}$ with symptoms; (v) severe aortic stenosis with a peak systolic pressure gradient greater than $50 \mathrm{~mm} \mathrm{Hg}$, with an aortic valve opening area less than $0.75 \mathrm{~cm}^{2}$; (vi) acute discomfort or fever; (vii) uncontrolled atrial or ventricular dysrhythmias; (viii) uncontrolled sinus rhythm tachycardia (more than 120 per minute); (ix) uncompensated congestive heart failure; (x) third degree atrioventricular block; (xi) acute pericarditis or myocarditis; (xii) recent embolism; (xiii) thrombophlebitis; (xiv) ST segment displacement is more than $2 \mathrm{~mm}$ at rest; and (xv) uncontrolled diabetes (glycemic blood glucose greater than $300 \mathrm{mg} / \mathrm{dL}$ or greater than 250 $\mathrm{mg} / \mathrm{dL}$ with ketone bodies). Afterwards, forty eligible PAD patients were randomly divided into general rehabilitation (GR) with CET (GR+CET, $n=20)$ and only received GR (GR, n=20) groups (Fig. S1). All subjects provided informed consent after the experimental procedures were explained. This study was performed in accordance with the tenets of the Declaration of Helsinki and approved by the Institutional Review Board of Chang Gung Memorial Hospital, Taiwan (ClinicalTrials.gov Identifier: NCT03965520).

\subsection{Ankle-brachial index (ABI) measurement}

Doppler measurements were performed in accordance with AHA guidelines for ABI measurement $[19,20]$. A digital vascular doppler HUNTLEIGH Dopplex DMX (Huntleigh Healthcare, United Kingdom) with an $8 \mathrm{MHz}$ probe was used to measure the individual systolic pressures. An appropriately sized pneumatic cuff was applied to the right upper arm, inflated to suprasystolic pressure and deflated slowly until a Doppler flow signal was detected. The process was repeated for right leg and values for both dorsal pedal and anterior tibial arteries were measured, followed by left leg and left arm. ABI was subsequently calculated for each lower limb separately using the value of pressure from the respective arm as a denominator. Moreover, we used duplex ultrasound scanning as the gold standard. DUS was performed using Vivid S6 Ultrasound System (GE Healthcare, USA) equipped with 8L-RS (a 5-13 MHz linear transducer) and 4C-RS (1.8-6 MHz curvilinear transducer).

\subsection{Grade exercise test}

The participants performed the graded exercise test on a bicycle ergometer (Ergoselect150P, Germany) by one rehabilitation physician who was blinded to the GR+CET 
or GR subjects to assess their cardiopulmonary parameters 2 days before and 2 days after 12week intervention. Moreover, the data collector was isolated from the data analytic specialist. Each participant was instructed to fast for at least $8 \mathrm{~h}$ and to refrain from exercise for at least $24 \mathrm{~h}$ before the test. All participants arrived at the testing center at 9:00 A.M. to eliminate diurnal effects. The subject first collected two minutes of resting parameters on the stationary bicycle. Then, the subjects performed on the unloaded free pedal for two minutes, after which the load was increased by 1 watt every 6 seconds ( 10 watts per minute) until the subjects were exhausted (i.e., progressive exercise to peak oxygen consumption, $\mathrm{VO}_{2 \text { peak }}$ ). During the process, the bicycle speed was required to be maintained at $60 \mathrm{rpm}$. Minute ventilation $\left(\mathrm{V}_{\mathrm{E}}\right), \mathrm{VO}_{2}$, and carbon dioxide production $\left(\mathrm{VCO}_{2}\right)$ were measured on a breath-by-breath basis, using a computer-based system (Master Screen CPX, Cardinal-health Germany) [14]. Heart rate (HR) was determined from the R-R interval on a 12 lead electrocardiogram, mean arterial pressure (MAP) was measured using an automatic blood pressure system (Tango, SunTech Medical, $\mathrm{UK}$ ), and arterial $\mathrm{O}_{2}$ saturation was monitored through finger pulse-oximetry (model 9500, Nonin Onyx, Plymouth, MN, USA) [14]. $\mathrm{VO}_{2 \text { peak }}$ was defined by the following criteria: $\mathrm{VO}_{2}$ increased by $<\mathrm{mL} / \mathrm{kg} / \mathrm{min}$ over at least $2 \mathrm{~min}$, $\mathrm{HR} \geq 85 \%$ of its predicted upper threshold, respiratory exchange ratio $\geq 1.10$, or some other symptom/sign limitations in accordance with the guidelines of the American College of Sports Medicine for exercise testing [21]. Additionally, the ventilation threshold (VT) was determined when $\mathrm{V}_{\mathrm{E}} / \mathrm{VO}_{2}$ increased without a corresponding increase in the $\mathrm{V}_{\mathrm{E}}$-to- $\mathrm{VCO}_{2}$ ratio, end-tidal $\mathrm{PO}_{2}$ increased without a decrease in end-tidal $\mathrm{PCO}_{2}$, or there was a departure from linearity for $\mathrm{V}_{\mathrm{E}}$ [21].

Ventilation and $\mathrm{VCO}_{2}$ responses, acquired from the initiation of exercise to the peak values, were used to calculate the $\mathrm{V}_{\mathrm{E}}-\mathrm{VCO}_{2}$ slope, using least-squares linear regression $(\mathrm{y}=\mathrm{m} \cdot \mathrm{x}+\mathrm{b}$, $\mathrm{m}=$ slope) [22]. Additionally, the 6-minute walk test (6 MWT) was used to assess functional capacity and exercise endurance in patients with PAD [23]. The distance covered over a time of 6 minutes was used as the outcome by which to compare changes in performance capacity [23].

\subsection{Hemodynamic measurements}

Noninvasive continuous cardiac output monitoring system (NICOM, Cheetah Medical, Wilmington, Delaware) was used to evaluate cardiac hemodynamic response to exercise, which analyzes the phase shift $(\Delta \Phi)$ created by alternating electrical current across the chest of the subject as described in our previous study [24]. Four dual surface electrodes were placed on the back of each subject to establish electrical contact with the body and avoid interference of upper body motion with the electrical cables during exercise testing. Stroke volume (SV) was estimated using the following equation: $\mathrm{SV}=\mathrm{C} \cdot \mathrm{VET} \cdot \mathrm{d} \Phi / \mathrm{dtmax}$, where $\mathrm{C}$ is a constant of proportionality, and VET denotes the ventricular ejection time, as determined using the NICOM and electrocardiogram signals. The cardiac output (CO) was then calculated using the following equation: $\mathrm{CO}=\mathrm{SV} \times \mathrm{HR}[24]$. 


\subsection{Cycling exercise training (CET) protocol}

In addition to the daily PAD rehabilitation course, the GR+CET subjects also performed supervised hospital-based training on a bicycle ergometer (Ergoselect 150P, Germany), completing 3 weekly sessions for 12 weeks. The CET protocol comprised a warm-up at 30\% of $\mathrm{VO}_{2 \text { peak }}$ for $3 \mathrm{~min}$, followed by continuous work-rate at $\mathrm{VT}$ for $30 \mathrm{~min}$, then a cool-down at $30 \%$ of $\mathrm{VO}_{2 \text { peak }}$ for $3 \mathrm{~min}$. All subjects used an HR monitor (Tango, SunTech Medical, UK) to obtain the assigned intensity of exercise. Borg 6-to-20 scale was used to assess the rate of perceived exertion during and after each exercise session. The work-rate of bicycle ergometer was adjusted continuously to ensure that the intensity of exercise matched the target HR throughout the training period. Patients were instructed to immediately stop exercise training if they had leg pain or other signs/symptoms of circulatory disorders. The GR subjects only engaged in general PAD rehabilitation course, as instructed by their rehabilitation physicians. The rates of compliance with the GR+CET and GR subjects were $100 \%$ and $100 \%$, respectively.

\subsection{Platelet isolation}

Before the graded exercise test at the beginning of the present study and 12 weeks later in various groups, $20 \mathrm{ml}$ of blood was sampled from each subject's antecubital vein within $1 \mathrm{~min}$ by venipuncture (20-gauge needle). Blood samples $(20 \mathrm{~mL})$ were collected in polypropylene tubes containing sodium citrate $(3.8 \mathrm{~g} / \mathrm{dL}, 1-9$ vol. blood). Platelet rich plasma (PRP) was prepared through centrifugation at $300 \mathrm{~g}$ for $10 \mathrm{~min}$ at approximately $20^{\circ} \mathrm{C}$. Platelets were sedimented through centrifugation of the PRP at $1500 \mathrm{~g}$ for $10 \mathrm{~min}$ at approximately $20^{\circ} \mathrm{C}$ and then washed once with phosphate buffered saline (PBS) containing ethylenediaminetetraacetic acid (EDTA, final concentration, $4 \mathrm{mM}$ ) (Sigma) to inhibit platelet activation $[13,14]$. The number of platelets was adjusted to $2 \times 10^{8}$ cells $/ \mathrm{mL}$ with mitochondrial respiration medium (MiR05, containing sucrose $110 \mathrm{mM}$, HEPES $20 \mathrm{mM}$, taurine $20 \mathrm{mM}$, K-lactobionate $60 \mathrm{mM}$, $\mathrm{MgCl}_{2} 3 \mathrm{mM}, \mathrm{KH}_{2} \mathrm{PO}_{4} 10 \mathrm{mM}$, EGTA $0.5 \mathrm{mM}$, BSA $1 \mathrm{~g} / \mathrm{L}, \mathrm{pH} 7.1$ ). Blood analysis was repeated twice to ensure reproducibility of the results. All platelet fractions were analyzed within $2 \mathrm{~h}$ after cell purification. Blood cells were enumerated using a Sysmax SF-3000 cell counter (GMI, Inc., Ramsey, MN, USA).

\subsection{High-resolution respirometry}

Platelet mitochondrial respiration was measured in $2 \mathrm{~mL}$ glass chambers of a highresolution respirometry (Oxygraph-2K, Oroboros Instrument, Austria) with a stirrer speed of $750 \mathrm{rpm}$ at a constant temperature of $37{ }^{\circ} \mathrm{C}$. Data was acquired and recorded every $2 \mathrm{~s}$ by DatLab software version 6 (Oroboros Instrument, Austria). The $\mathrm{O}_{2}$ sensors and instrument background $\mathrm{O}_{2}$ consumption had been already calibrated before experiments following the manufacturer's instruction $[13,14]$. Fixed number of platelets $\left(2 \times 10^{8}\right.$ cells $\left./ \mathrm{mL}\right)$ were added in the glass chamber filled with $2 \mathrm{~mL}$ mitochondrial respiration medium (MiR05) for measurement $[13,14]$. Air calibration at saturation point was performed each time before the 
experiment for quality control. $\mathrm{O}_{2}$ concentration was automatically calculated from barometric pressure and solubility factor was 0.92 for MiR05.

\subsection{Mitochondrial ETS and OXPHOS in platelets}

A documented protocol, the substrate-uncoupler-inhibitor titration reference protocol (SUIT-RP), was applied, consisting of two harmonized mitochondrial substrate-controlled experiments (RP1 and RP2) to acquire most information (Figs. S2A-S2D) [18]. All chemicals were purchased from Sigma-Aldrich (St. Louis, MO), if not stated otherwise.

RP1 is the SUIT experiment to measure mainly the capacity of mitochondrial ETS (Figs. S2A and S2B). Routine respirations of platelets were obtained after the cells stabilized in the $\mathrm{O} 2 \mathrm{~K}$ chamber. The respirations of intact cells were driven by endogenous substrates in this state. To access the mitochondrial respiration with saturating exogenous substrates, the cell membrane was then permeabilized with digitonin [18]. The concentrations of digitonin titration were $50 \mu \mathrm{g} / 4 \times 10^{8}$ platelets. After cell permeabilization, concomitantly added NADH-linked (N-linked) substrates malate $(2 \mathrm{mM})$ and pyruvate $(5 \mathrm{mM})$. Because of absent in ADP, the oxygen consumption was only driven by uncoupling proton leakage (LEAK state, $\mathrm{PM}_{\mathrm{L}}$ ). The oxidative phosphorylation (OXPHOS) capacity $\left(\mathrm{PM}_{\mathrm{P}}\right)$ was then evaluated by ADP (Calbiochem, USA) titration $(1 \mathrm{mM})$. A cytochrome $\mathrm{C}(10 \mu \mathrm{M})$ test was applied to check if any disruption of the mitochondrial outer membrane and loss of cytochrome $\mathrm{c}$ during the process of cell permeabilization. The ETS capacity driven by malate and pyruvate $\left(\mathrm{PM}_{\mathrm{E}}\right)$ was subsequently obtained by mitochondrial protonophore carbonyl cyanide-ptrifluoromethoxyphenylhydrazone (FCCP) titration $(0.5 \mu \mathrm{M} / \mathrm{step})$ until no further respiration increase. Glutamate $(10 \mathrm{mM})$ was added to fulfill the N-linked substrates, accessing the maximal ETS capacity driven by NADH or complex I (CI)-related resources (MPGE). $10 \mathrm{mM}$ succinate was added to complete the convergent input of CI and complex II (CII) (MPGS $\mathrm{E}_{\mathrm{E}}$ ), followed by an octanoyl-carnitine titration $(0.5 \mathrm{mM})$ to check the additional effect of fatty acid oxidation (MPGSOct $)$. Mitochondrial CI inhibitor, rotenone $(0.5 \mu \mathrm{M})$, was added to block the respiration from $\mathrm{N}$-linked substrates dependent and fatty acid oxidation pathways $\left(\mathrm{S}_{\mathrm{E}}\right)$. The additional contribution of the mitochondrial complex of glycerophosphate dehydrogenase $(\mathrm{CGpDH})$ was evaluated with $10 \mathrm{mM}$ glycerophosphate addition ( $\mathrm{SG}_{\mathrm{E}}$ ). At last, the mitochondrial respiration was inhibited by antimycin $\mathrm{A}(2.5 \mu \mathrm{M})$, the mitochondrial complex III inhibitor.

The capacities of mitochondrial ETS in permeabilized platelets were calculating from the results of RP1 protocol using the following equations:

$\mathrm{ETS}_{\mathrm{CI}}\left(\mathrm{PMG}_{\mathrm{E}}\right)=$ Pyruvate + Malate $+\mathrm{ADP}+\mathrm{FCCP}+$ Glutamate .................. [1]

$\mathrm{ETS}_{\mathrm{CI}+\mathrm{CII}}\left(\mathrm{PMGS}_{\mathrm{E}}\right)=$ Pyruvate + Malate $+\mathrm{ADP}+\mathrm{FCCP}+$ Glutamate + Succinate ... [2]

$\mathrm{ETS}_{\mathrm{CI}+\mathrm{CII}+\mathrm{FAO}}(\mathrm{PMGSOct})=$ Pyruvate + Malate $+\mathrm{ADP}+\mathrm{FCCP}+$ Glutamate + Succinate

+ Octanoyl-Carnitine 
ETS $_{\text {CII }}\left(\mathrm{S}_{\mathrm{E}}\right)=$ Pyruvate + Malate + ADP + FCCP + Glutamate + Succinate + OctanoylCarnitine + Rotenone

ETS $_{\mathrm{CII}+\mathrm{Gp}}\left(\mathrm{SGp}_{\mathrm{E}}\right)=$ Pyruvate + Malate $+\mathrm{ADP}+\mathrm{FCCP}+$ Glutamate + Succinate +

Octanoyl-Carnitine + Rotenone + Glycerophosphate

RP2 is another SUIT experiment to measure mainly the OXPHOS state (ATP synthase dependent) of mitochondrial respiration (Figs. S2C and S2D). Following routine respiration and digitonin permeabilization, $1 \mathrm{mM}$ ADP was titrated to accelerate the depletion of residual endogenous substrates. Afterwards, octanoyl-carnitine $(0.5 \mathrm{mM})$ and malate $(0.1 \mathrm{mM})$ were added to evaluated the fatty acid oxidation. High malate concentration $(2 \mathrm{mM})$ was required to reduce the flux from succinate dehydrogenase, but could also activate the mitochondrial malic enzyme and led to higher $\mathrm{O}_{2}$ consumption $\left(\mathrm{OctM}_{\mathrm{P}}\right)$. Cytochrome $\mathrm{C}$ test was applied after high malate titration, to ensure the quality and intactness of mitochondria. $5 \mathrm{mM}$ pyruvate (OctMP $\left.\mathrm{P}_{\mathrm{P}}\right)$ and $10 \mathrm{mM}$ glutamate $\left(\mathrm{OctMPG}_{\mathrm{P}}\right)$ were sequentially titrated to acquire the N-linked respiration. A consecutive titration of succinate $(10 \mathrm{mM})$ and glycerophosphate $(10 \mathrm{mM})$ stimulated the

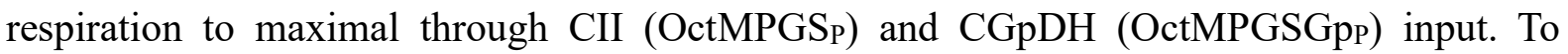
evaluate the limitation of OXPHOS system, FCCP $(0.5 \mu \mathrm{M} /$ step $)$ is titrated to access the maximum ETS capacity (OctMPGSGp $\mathrm{E}_{\mathrm{E}}$ ). $0.5 \mu \mathrm{M}$ rotenone was added to reach the identical $\mathrm{SGp}_{\mathrm{E}}$ state in RP1 and RP2, and linked these two protocols for harmonization. Antimycin A $(2.5 \mu \mathrm{M})$ was added in final to block the whole respiration.

The capacity of mitochondrial OXPHOS in permeabilized platelets were calculating from the results of RP2 protocol using the following equations:

$\operatorname{OXPHOS}_{\mathrm{FAO}}\left(\right.$ OctP $\left._{\mathrm{P}}\right)=\mathrm{ADP}+$ Octanoyl-Carnitine

$\mathrm{OXPHOS}_{\mathrm{FAO}+\mathrm{CI}}($ OctMPGP $)=\mathrm{ADP}+$ Octanoyl-Carnitine + Malate + Pyruvate +

Glutamate

$\mathrm{OXPHOS}_{\mathrm{FAO}+\mathrm{CI}+\mathrm{CII}}($ OctMPGSP $)=\mathrm{ADP}+$ Octanoyl-Carnitine + Malate + Pyruvate +

Glutamate + Succinate

This study defines the maximal capacities of OXPHOS and ETS in platelets as the OctMPGSGp $_{P}$ and OctMPGSGp states in RP2 protocol, respectively (Fig. S2B).

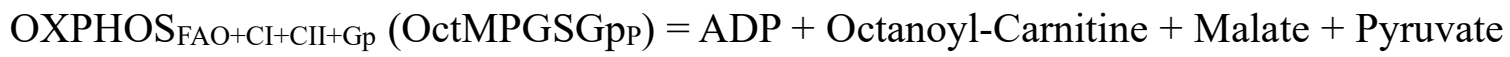

+ Glutamate + Succinate + Glycerophosphate

$\mathrm{ETS}_{\mathrm{FAO}+\mathrm{CI}+\mathrm{CII}+\mathrm{Gp}}($ OctMPGSGp $)=\mathrm{ADP}+$ Octanoyl-carnitine + Malate + Pyruvate +

Glutamate + Succinate + Glycerophosphate + FCCP

\subsection{Health-related quality of life}

Health-related quality of life (HRQoL) in the PAD population were measured using the Short Form-36 Health Survey questionnaire (SF-36). SF-36 is a generic measure and can help differentiate QoL issues related to co-morbidities from those related to PAD [25]. 


\subsection{Statistical analysis}

Data were expressed as mean \pm SEM and were analyzed using the statistical software package StatView. Experimental results were analyzed by 2 (groups) $\times 2$ (time sample points; i.e., pre- and post-interventions) repeated measures ANOVA with Bonferroni's post hoc test to compare cardiopulmonary fitness and capacities of mitochondrial OXPHOS and ETS in platelets at the beginning of this study and after 12 weeks in the GR+CET and GR groups. Pearson correlation analysis was used to determine the association between aerobic capacity (i.e., $\mathrm{VO}_{2 \text { peak }}$ ) and platelet mitochondrial OXPHOS and ETS in patients with PAD. The criterion for significance was $P<0.05$. 


\section{Results}

\subsection{Cardiopulmonary fitness and HRQoL}

Fifty-two patients diagnosed with PAD have been surveyed for the interventions. A total of 40 eligible subjects completed the study in the GR+CET $(n=20)$ and GR $(n=20)$ groups. No adverse vascular or thrombotic event occurred in the two groups throughout the periods of investigation (Fig. 1S). Moreover, both GR+CET and GR groups did not differ significantly in anthropometric and clinical parameters (Table 1) or cardiopulmonary fitness (Table 2) at the start of the study.

Following 12 weeks of interventions, the GR+CET group increased values of ABI at right $(\mathrm{R})$ and left $(\mathrm{L})$ limbs using Duplex ultrasonography ( $\mathrm{R}$ values from 0.85 to 0.92 ; $\mathrm{L}$ values from 0.86 to $0.93, P<0.05$ ) and sphygmometry (R values from 0.86 to 0.93 ; $\mathrm{L}$ values from 0.87 vs. $0.94, P<0.05$ ), respectively (Table 1 ). In cardiopulmonary fitness, 12-week GR+CET also increased work-rate, $\mathrm{HR}, \mathrm{SV}, \mathrm{CO}$, as well as, $\mathrm{V}_{\mathrm{E}}, \mathrm{VO}_{2}$, and $\mathrm{VCO}_{2}$ at the peak exercise performance $(P<0.05)$, but no significant changes in aerobic capacity occurred in the GR group (Table 2). Moreover, the GR+CET regimen significantly increased the distance in 6 MWT (initial pain from $217.6 \mathrm{~m}$ to $323.5 \mathrm{~m}$; terminal point from $363.0 \mathrm{~m}$ to $451.0 \mathrm{~m}, P<0.05$ ) and MET (scores from 3.52 to 5.14, $P<0.05$ ), as well as, lowered $\mathrm{V}_{\mathrm{E}}-\mathrm{VCO}_{2}$ slope (values from 38.8 to $34.2, P<0.05$ ) in the PAD patients (Table 2). Conversely, the GR alone did not influence the values of these indices of ventilatory efficiency and functional capacity in patients with PAD (Table 2).

In HRQoL, the GR+CET for 12 weeks significantly increased the subclass scores of the physical (scores from 37.1 to $43.1, P<0.05$ ) and mental (scores from 35.2 to $42.8, P<0.05$ ) dimensions in SF-36, respectively (Table 2). However, the GR alone remained unchanged the scores of SF-36 physical and mental components (Table 2).

\subsection{Blood cell count}

There were no significant changes in erythrocyte, hemoglobin, hematocrit, lymphocyte and platelet following 12 weeks of the GR with or without CET (Table 3). However, the GR + CET considerably decreased neutrophil count (counts from 5.30 to $3.78 \times 10^{3} / \mu 1, P<0.05$ ) and the ratio of neutrophils to lymphocytes (NLR, ratio from 3.43 to $2.22, P<0.05$ ). Moreover, these blood cell counts were unchanged following the GR alone (Table 3).

\subsection{Mitochondrial ETS and OXPHOS in platelets}

Either GR+CET or GR for 12 weeks unchanged the values of routine respiration and uncoupling proton leakage of platelets (Figs. 1 and 2). However, the GR with CET significantly increased FADH2 (CII)-related ETS levels in platelet $\left(\mathrm{PMGS}_{\mathrm{E}}, \mathrm{PMGSOct}_{\mathrm{E}}, \mathrm{S}_{\mathrm{E}}\right.$, and $\mathrm{SG}_{\mathrm{E}}$ ) (Fig. 1A, $P<0.05)$. Additionally, the GR+CET enhanced succinate-involved OXPHOS level in platelet, such as OctMPGS (Fig. 2A, $P<0.05$ ) rather than OctMPGP. Furthermore, this exercise regimen also augmented the capacities of maximal OXPHOS (OctMPGSGpP, from 76.1 to 
101.2 pmol $/ \mathrm{s} / 10^{8}$ cells, $P<0.05$ ) and ETS (OctMPGSGp , from 106.1 to $155.3 \mathrm{pmol} / \mathrm{s} / 10^{8}$ cells, $P<0.05$ ) in platelet, respectively (Fig. 2A). Nevertheless, no significant changes in platelet mitochondrial bioenergetics, that included ETS and OXPHOS capacities, occurred following GR alone (Figs. 1B and 2B).

On the other hand, changes of $\mathrm{VO}_{2 \text { peak }}$ levels were positively associated with changes of maximal OXPHOS (OctMPGSGpP) (Fig.3A, r=0.681, $P<0.001$ ) and ETS (OctMPGSGp ) (Fig. 3B, r=0.706, $P<0.001$ ) in platelets, respectively. 


\section{Discussion}

This investigation clearly exhibits that 12 -week GR+CET improves systemic aerobic capacity and ventilatory/hemodynamic efficiencies, which is accompanied by improving health-related quality of life in patients with PAD. Notably, this study is the first to demonstrate that the CET regimen effectively enhances capacities of mitochondrial OXPHOS and ETS in platelet through increasing Complex II activity in PAD patients.

\subsection{Ventilation/hemodynamic efficiencies and HRQoL}

Optimal exercise programs increase the ability of PAD patients to independently perform activities of daily living, thereby further improving their quality of life [2,3]. In this study, 12week $\mathrm{GR}+\mathrm{CET}$ increased $\mathrm{VO}_{2 \text { peak }}$ and decreased $\mathrm{V}_{\mathrm{E}}-\mathrm{VCO}_{2}$ slope, as well as, enhanced hemodynamic responses (such as $\mathrm{CO}$ and $\mathrm{SV}$ ) to exercise in the PAD patients. The ventilatory parameters obtained from the graded exercise test may convey information regarding prognosis of circulatory disorders [22]. The $\mathrm{VO}_{2 \text { peak }}$ is an indicator of aerobic capacity, respectively [22], whereas the $\mathrm{V}_{\mathrm{E}}-\mathrm{VCO}_{2}$ slope is a powerful predictor of survival in patients with circulatory disorders [22]. Our previous study using patients with HF reported that these indices of ventilatory efficiency modulated by exercise training were correlated with exercise-induced central and peripheral hemodynamic changes [24]. Accordingly, the GR+CET may effectively improve ventilatory and hemodynamic efficiencies, thereby enhancing systemic aerobic capacity in patients with PAD.

Beside an improvement in systemic aerobic capacity, CET for 12 weeks also increased the distance in 6-minute walk test and consequently heightened the Short Form-36 physical/mental component scores. These findings imply that the exercise regimen effectively enhances the ability of patients to cope with the physical demands of daily activity and subsequently improving psychosocial status in PAD patients. Furthermore, the better HRQoL might exhibit less potential for mortality in PAD patients and simultaneously reduce the financial burden in their health care system $[2,3]$.

\subsection{Mitochondrial functionalities in platelets}

Progression to PAD is probably associated with a gradual decline in bioenergetic reserve capacity owing to the inability of endogenous homeostatic mechanisms to compensate for the insufficient energy supply [26]. Moreover, mitochondrial dysfunction in PAD patients may result in systemic inflammation that facilitates susceptibility to energy-based pathologies associated with oxidative stress [27]. Additionally, atherosclerosis and thrombosis are reported to be associated with deterioration of platelet mitochondrial function [6,7]. Hence, it is plausible that platelet mitochondrial bioenergetics is a marker for metabolic stress in PAD progression.

NLR has been associated with systemic inflammation and atherosclerotic burden, leading to inferior outcomes after lower extremity interventions [28]. Mitochondria are highly sensitive 
to inflammatory stress and respond dynamically to changes in their microenvironment [29]. Decreased oxidative capacity due to mitochondrial respiratory chain impairment may be associated with increased release of reactive oxygen species (ROS) and reduction of calcium retention capacity, leading to enhanced apoptosis of skeletal muscles [30]. The present investigation further demonstrated that CET significantly depressed circulatory inflammatory status, indicated by decreased neutrophil count and NLR in patients with PAD. Hence, we posit that the CET-induced physiological adaptations protect against inflammation associated with mitochondrial dysfunction in PAD patients [31].

A previous study reported that ischemia results in accumulation of intracellular succinate, thus leading to elevated mitochondrial ROS production [32]. Elevation of oxidative stress may also induce succinate accumulation by decreasing SDH activity [33]. Recently, our study including sedentary healthy men reported that CET significantly enhanced platelet SDH activity and Complex II-related respiration following hypoxic stress [13]. In clinical investigation, we further demonstrated that CET elevated platelet mitochondrial $\mathrm{O}_{2}$ consumption rate via increasing Complex II activity, which was accompanied by depressed oxidative stress in patients with HF [14]. In the present study, 12-week CET effectively also enhanced platelet mitochondrial OXPHOS and ETS capacities by increasing Complex II activity in the PAD patients. Therefore, CET-induced elevation in Complex II activity in platelets may rapidly eliminate succinate, further reducing ROS production from platelet mitochondria, eventually depressing circulatory oxidative stress and pro-inflammatory status in patients with $\mathrm{PAD}$. Moreover, we further demonstrate that change of $\mathrm{VO}_{2 \text { peak }}$ was positively correlated with changes of maximal OXPHOS and ETS capacities in platelets. These experimental results suggest that CET-induced platelet metabolic adaptation may be associated with improved systemic aerobic capacity in patients with PAD.

\subsection{Limitations of this study}

Our small sample size in each group is a major limitation. However, results regarding platelet mitochondrial bioenergetics have high values of statistical power ranging from 0.864 to 1.000. Additionally, this study mainly focused on the effects of CET on platelet mitochondrial bioenergetics rather than platelet reactivity (adhesion and aggregation) or platelet-mediated coagulation or thrombin generation. Our previous studies have investigated the effect of CET on platelet adhesiveness and aggregation and their underling mechanisms in healthy people and patients with cardiovascular disorders [9-12]. Recent study further reported that CET markedly suppressed hypoxia-induced oxidative damage of platelet mitochondria and consequent attenuation of platelet-mediated thrombin generation caused by hypoxia in healthy sedentary men [13]. However, the role of platelet mitochondrial function on CET-mediated platelet reactivity and coagulation in PAD patients warrants further investigation.

\subsection{Conclusions}


In the present study, 12-week CET enhances systemic aerobic capacity through enhancing ventilatory/hemodynamic efficiencies, as well as improved HRQoL in patients with PAD. Simultaneously, the exercise regimen also increased the capacities of OXPHOS and ETS by enhancing mitochondrial Complex II activity in platelets. These experimental findings may facilitate the identification of effective exercise regimen to increase physical performance and improve the efficacy for platelet mitochondrial bioenergetics in PAD patients. 


\section{Author contributions}

Jong-Shyan Wang, Tieh-Cheng $\mathrm{Fu}$, and Chih-Chin Hsu was involved in conception and design of research; Ming-Lu Lin, Tieh-Cheng Fu, Shu-Chun Huang, and Yu-Ting Lin performed experiments; Jong-Shyan Wang, Tieh-Cheng Fu and Ming-Lu Lin analyzed data, interpreted results of experiments, prepared the Figures and drafted the paper; Jong-Shyan Wang and Tieh-Cheng Fu edited and revised the paper; Jong-Shyan Wang, Ming-Lu Lin, TiehCheng Fu, Chih-Chin Hsu, Shu-Chun Huang, and Yu-Ting Lin approved the final version of paper.

\section{Acknowledgments}

The authors would like to thank the volunteers for their enthusiastic participation.

\section{Funding}

This work was supported by the National Science Council of Taiwan (grant number NSC 180-2314-B-182-039-MY3) and Chang Gung Medical Research Program (grant number CMRPD1J0221).

\section{Conflicts of interest}

No conflicts of interest, financial or otherwise, are declared by the authors. 


\section{References}

1. Kithcart, A.P.; Beckman, J.A. ACC/AHA versus ESC guidelines for diagnosis and management of peripheral artery disease: JACC Guideline Comparison. J. Am. Coll. Cardiol. 2018, 72, 2789-2801.

2. Treat-Jacobson, D.; McDermott, M.M.; Bronas U.G. et al. (2019) Optimal exercise programs for patients with peripheral artery disease: A scientific statement From the American Heart Association. Circulation 2019, 139, e10-e33.

3. Treat-Jacobson, D.; McDermott, M.M.; Beckman, J.A. et al. Implementation of supervised exercise therapy for patients with symptomatic peripheral artery disease: a science advisory from the American Heart Association. Circulation 2019, 140, e700-e710.

4. Klonaris. C.; Patelis. N.; Drebes. A.; Matheiken. S.; Liakakos, T. Antiplatelet treatment in peripheral arterial disease: the role of novel antiplatelet agents. Curr. Pharm. Des. 2016, 22, 4610-4616.

5. Tsigkou. V.; Siasos. G.; Rovos, K.; Tripyla, N.; Tousoulis, D. Peripheral artery disease and antiplatelet treatment. Curr. Opin. Pharmacol. 2018, 39, 43-52.

6. Garcia-Souza, L.F.; Oliveira, M.F. Mitochondria: biological roles in platelet physiology and pathology. Int. J. Biochem. Cell Biol. 2014, 50, 156-160.

7. Zharikov, S.; Shiva, S. Platelet mitochondrial function: from regulation of thrombosis to biomarker of disease. Biochem. Soc. Trans. 2013, 41, 118-123.

8. Kramer, P.A.; Chacko, B.K.; Ravi, S.; Johnson, M.S.; Mitchell, T.; Darley-Usmar, V.M. Bioenergetics and the oxidative burst: Protocols for the isolation and evaluation of human leukocytes and platelets. J. Vis. Exp. 2014, 85, e51301.

9. Wang, J.S. Exercise prescription and thrombogenesis. J. Biomed. Sci. 2006, 13, 753-761.

10. Wang, J.S.; Jen, C.J.; Kung, H.C.; Lin, L.J.; Hsiue, T.R.; Chen, H.I. Different effects of strenuous exercise and moderate exercise on platelet function in men. Circulation 1994, 90, 2877-2885.

11. Wang, J.S.; Jen, C.J.; Chen, H.I. Effects of exercise training and deconditioning on platelet function in men. Arterioscler. Thromb. Vasc. Biol. 1995, 15, 1668-1674.

12. Wang, J.S.; Li, Y.S.; Chen, J.C. Effects of exercise training and deconditioning on platelet aggregation induced by alternating shear stress in men. Arterioscler. Thromb. Vasc. Biol. 2005, 25, 454-460.

13. Wu, L.H.; Chang, S.C.; Fu, T.C.; Huang, C.H.; Wang, J.S. High-intensity interval training improves mitochondrial function and suppresses thrombin generation in platelets undergoing hypoxic stress. Sci. Rep. 2017, 7, e4191.

14. Chou, C.H.; Fu, T.C.; Tsai, H.H.; Hsu, C.C.; Wang, C.H.; Wang, J.S. High-intensity interval training enhances mitochondrial bioenergetics of platelets in patients with heart failure. Int. 
J. Cardiol. 2019, 274, 214-220.

15. Letts, J.A.; Fiedorczuk, K.; Sazanov, L.A. The architecture of respiratory supercomplexes. Nature 2016, 537, 644-648.

16. Picard, M.; Taivassalo, T.; Ritchie, D.; Wright, K.J.; Thomas, M.M.; Romestaing, C.; Hepple, R.T. Mitochondrial structure and function are disrupted by standard isolation methods. PLoS. One 2011, 6, e18317.

17. Pesta, D.; Gnaiger, E. High-resolution respirometry: OXPHOS protocols for human cells and permeabilized fibers from small biopsies of human muscle. Methods Mol. Biol. 2012, 810, 25-58.

18. Hsu, C.C.; Tsai, H.H.; Fu, T.C.; Wang, J.S. Exercise training enhances platelet mitochondrial bioenergetics in stroke patients: a randomized controlled trial. J. Clin. Med. 2019, 8, 2186.

19. Misra, S.; Shishehbor, M.H.; Takahashi, E.A. et al. Perfusion assessment in critical limb ischemia: principles for understanding and the development of evidence and evaluation of devices: a scientific statement from the American Heart Association. Circulation 2019, 140, e657-e672.

20. Guirguis-Blake, J.M.; Evans, C.V.; Redmond, N.; Lin, J.S. Screening for peripheral artery disease using the ankle-brachial index: updated evidence report and systematic review for the US preventive services task force. JAMA 2018, 320, 184-196.

21. American College of Sports Medicine. General principle of exercise prescription. In ACSM's Guidelines for Exercise Testing and Prescription (Thompson, W. R., Gordon, N. F. and Pescatello, L. S., eds), Lippincott Williams \& Wilkins, Philadelphia 2010, pp. 152182.

22. Arena, R.; Guazzi, M.; Myers, J. Ventilatory abnormalities during exercise in heart failure: a mini review. Curr. Respir. Med. Rev. 2007, 3, 179-187.

23. ATS Committee on Proficiency Standards for Clinical Pulmonary Function Laboratories. ATS statement: guidelines for the six-minute walk test. Am. J. Respir. Crit. Care Med. 2002, $166,111-117$.

24. Fu, T.C.; Wang, C.H.; Lin, P.S.; Hsu, C.C.; Cherng, W.J.; Huang, S.C.; Liu, M.H.; Chiang, C.L.; Wang, J.S. Aerobic interval training improves oxygen uptake efficiency by enhancing cerebral and muscular hemodynamics in patients with heart failure. Int. J. Cardiol. 2013, 167, 41-50.

25. Maksimovic, M.; Vlajinac, H.; Marinkovic, J.; Kocev, N.; Voskresenski, T.; Radak, D. Health-related quality of life among patients with peripheral arterial disease. Angiology 2014, 65, 501-506.

26. Rontoyanni, V.G.; Nunez Lopez, O.; Fankhauser, G.T.; Cheema, Z.F.; Rasmussen, B.B.; Porter, C. Mitochondrial bioenergetics in the metabolic myopathy accompanying 
peripheral artery disease. Front. Physiol. 2017, 8, 141.

27. Koutakis, P.; Ismaeel, A.; Farmer, P.; Purcell, S.; Smith, R.S.; Eidson, J.L.; Bohannon, W.T. Oxidative stress and antioxidant treatment in patients with peripheral artery disease. Physiol. Rep. 2018, 6, e13650.

28. Teperman, J.; Carruthers, D.; Guo, Y.; Barnett, M.P.; Harris, A.A.; Sedlis, S.P.; Pillinger, M.; Babaev, A.; Staniloae, C.; Attubato, M.; Shah, B. Relationship between neutrophillymphocyte ratio and severity of lower extremity peripheral artery disease. Int. J. Cardiol. 2017, 228, 201-204.

29. Carter, H.N.; Chen, C.C.; Hood, D.A. Mitochondria, muscle health, and exercise with advancing age. Physiology (Bethesda) 2015, 30, 208-223.

30. Anzell, A.R.; Maizy, R.; Przyklenk, K.; Sanderson, T.H. Mitochondrial quality control and disease: insights into ischemia-reperfusion injury. Mol. Neurobiol. 2018, 55, 2547-2564.

31. Pizzimenti, M.; Riou, M.; Charles, A.L.; Talha, S.; Meyer, A.; Andres, E.; Chakfé, N.; Lejay, A.; Geny, B. The rise of mitochondria in peripheral arterial disease physiopathology: experimental and clinical data. J. Clin. Med. 2019, 8, 2125.

32. Chouchani, E.T.; Pell, V.R.; Gaude, E. et al. Ischaemic accumulation of succinate controls reperfusion injury through mitochondrial ROS. Nature 2014, 515, 431-435.

33. Mills, E.; O'Neill, L.A. Succinate: a metabolic signal in inflammation. Trends Cell. Biol. 2014, 24, 313-320. 


\section{FIGURE LEGENDS}

Figure 1: Effects of general rehabilitation with $(\mathbf{A}, \mathbf{G R}+\mathbf{C E T})$ and without $(\mathbf{B}, \mathbf{G R})$ cycling exercise training in platelet mitochondrial $\mathrm{O}_{2}$ consumption rate (OCR) using the reference protocol 1 (RP1) protocol in patients with peripheral arterial disease. P, pyruvate; M, malate; G, glutamate; S, succinate; Oct, octanoyl-carnitine; Gp, glycerophosphate; Rot, rotenone; Ama, antimycin; ROX, residual $\mathrm{O}_{2}$ consumption; OXPHOS or P, oxidative phosphorylation; ETS or E, electron transport system; LEAK or L, uncoupling proton leakage. Pre-intervention vs. Post-intervention, * $P$ $<0.05$. Values were mean \pm SEM.

Figure 2: Effects of general rehabilitation with $(\mathbf{A}, \mathbf{G R}+\mathbf{C E T})$ and without $(\mathbf{B}, \mathbf{G R})$ cycling exercise training in platelet mitochondrial $\mathrm{O}_{2}$ consumption rate (OCR) using the reference protocol 2 (RP2) protocol in patients with peripheral arterial disease. $\mathbf{P}$, pyruvate; M, malate; G, glutamate; S, succinate; Oct, octanoyl-carnitine; Gp, glycerophosphate; Rot, rotenone; Ama, antimycin; ROX, residual $\mathrm{O}_{2}$ consumption; OXPHOS or P, oxidative phosphorylation; ETS or E, electron transport system; LEAK or $\mathrm{L}$, uncoupling proton leakage. Pre-intervention vs. Post-intervention, ${ }^{*} P$ $<0.05$. Values were mean \pm SEM.

Figure 3: Correlations between changes of systemic aerobic capacity $\left(\mathrm{VO}_{2 \text { peak }}\right)$ and platelet maximal (A) oxidative phosphorylation (OXPHOS) and (B) electron transport system (ETS) capacities in patients with peripheral arterial disease. (A) maximal OXPHOS capacity (OctMPGSGpp) $=$ ADP + Octanoyl-Carnitine + Malate + Pyruvate + Glutamate + Succinate + Glycerophosphate; (B) maximal ETS capacity $($ OctMPGSGp $)=$ ADP + Octanoyl-carnitine + Malate + Pyruvate + Glutamate + Succinate + Glycerophosphate + FCCP. 


\section{SUPPLEMENTARY MATERIALS}

Figure S1: Flowchart of enrolled patients with peripheral arterial disease (PAD) during following-up. Inclusion and exclusion criteria listed in the figure were used to recruit eligible candidates. Recruited PAD patients were divided into two groups based on their treatments: general rehabilitation with $(\mathbf{G R}+\mathbf{C E T})$ and without $(\mathbf{G R})$ cycling exercise training. In addition to general rehabilitation, the GR+CET group performed supervised hospital-based training on a bicycle ergometer (i.e., cycling exercise at ventilation threshold for $30 \mathrm{~min} /$ day, 3 days/week for 12 weeks). The GR group only engaged in general rehabilitation course for 12 weeks, as instructed by their rehabilitation physicians.

Figure S2: Graphs showing a sample of cycling exercise training (CET) effects on platelet mitochondrial oxidative phosphorylation (OXPHOS) (A, B) and electron transport system (ETS) (C, D) in a patient with peripheral arterial disease. The reference protocols $[\mathbf{R P 1}(\mathbf{A}, \mathbf{B})$ and $\mathbf{R P 2}(\mathbf{C}, \mathbf{D})]$ are the substrate-uncoupler-inhibitor titration (SUIT) experiments to measure mainly the capacities of mitochondrial oxidative phosphorylation (OXPHOS) (A, B) and electron transport system (ETS) $(\mathbf{C}, \mathbf{D})$ in platelets, respectively. Pre-GR+CET, before general rehabilitation with cycling exercise training; Post-GR+CET, after general rehabilitation with cycling exercise training; D, digoxin; P, pyruvate; M, malate; ADP, adenosine diphosphate; Cyt C, cytochrome C; FCCP, carbonyl cyanide-4-(trifluoromethoxy) phenylhydrazone; G, glutamate; S, succinate; Oct, octanoyl-carnitine; Rot, rotenone; Gp, glycerophosphate; Ama, antimycin; FAO, fatty acid oxidation; LEAK, uncoupling proton leakage; OXPHOS, oxidative phosphorylation; ETS, electron transport system. 
Fig. 1

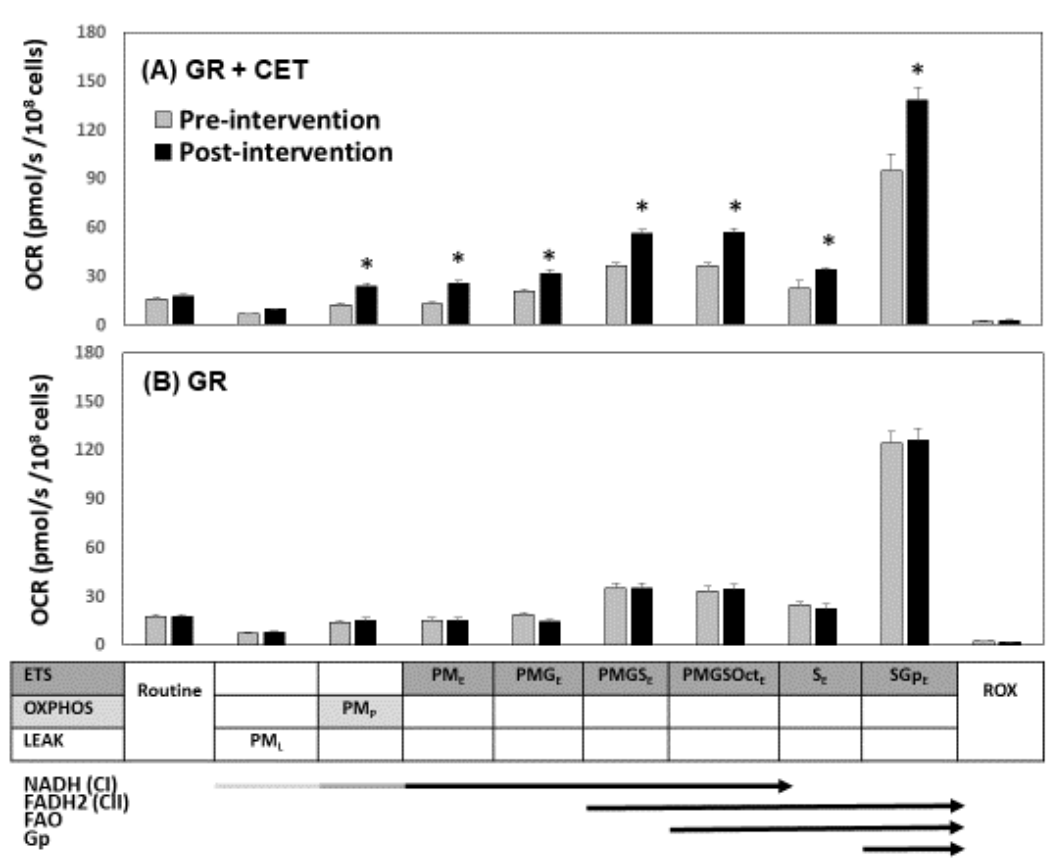


Fig. 2

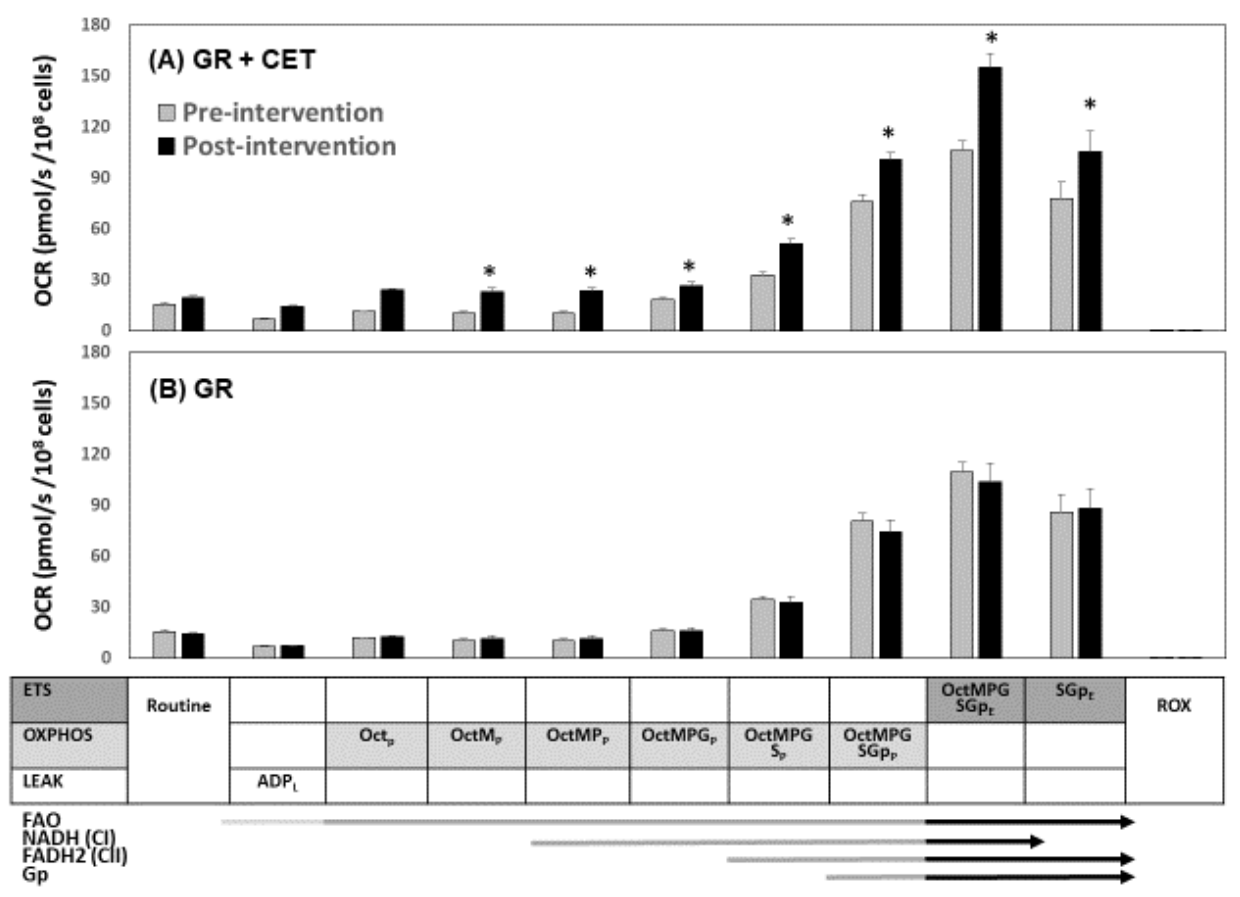


Fig. 3

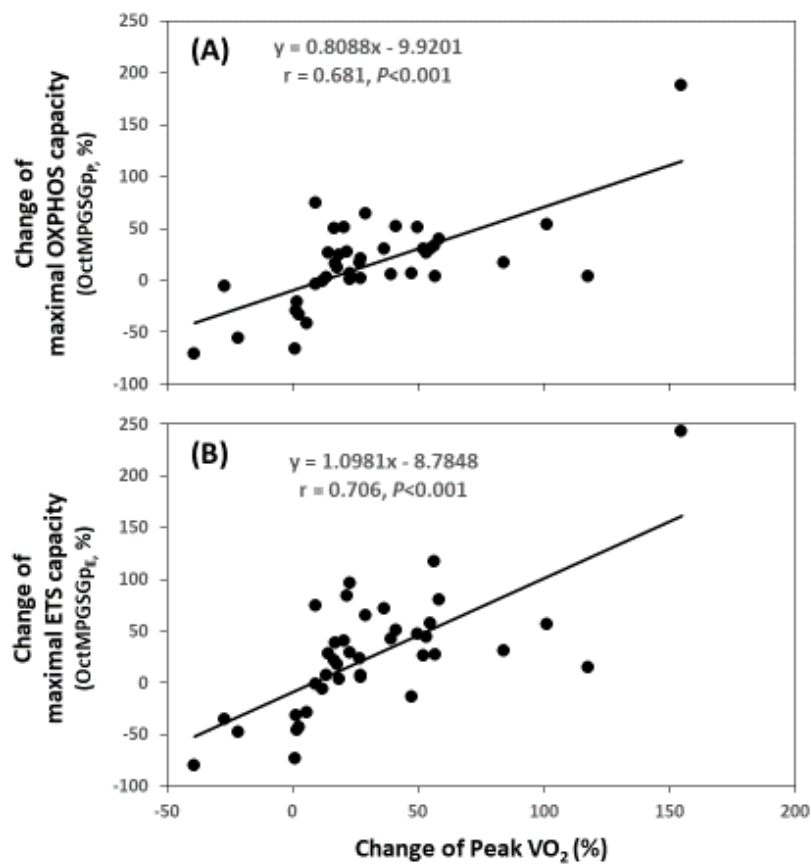


Table 1: Demographic and clinical characteristics in patients with peripheral arterial disease

\begin{tabular}{|c|c|c|c|c|c|}
\hline & \multicolumn{2}{|c|}{ GR+CET } & \multicolumn{2}{|c|}{ GR } \\
\hline & & Pre & Post & Pre & Post \\
\hline \multicolumn{6}{|c|}{ Anthropometrics/Clinical Characteristics } \\
\hline Gender & $\mathrm{n}(\mathrm{M} / \mathrm{F})$ & $20(12 / 8)$ & $20(12 / 8)$ & $20(12 / 8)$ & $20(12 / 8)$ \\
\hline Age & year & $71.1 \pm .1 .5$ & - & $70.5 \pm .1 .9$ & - \\
\hline Height & $\mathrm{cm}$ & $160.2 \pm 1.3$ & - & $159.0 \pm 2.0$ & $166.8 \pm 1.5$ \\
\hline Weight & $\mathrm{kg}$ & $69.7 \pm 2.4$ & $69.3 \pm 2.4$ & $67.6 \pm 4.0$ & $67.3 \pm 3.9$ \\
\hline Heart rate & bpm & $81 \pm 2$ & $81 \pm 2$ & $80 \pm 2$ & $81 \pm 3$ \\
\hline Systolic blood pressure & $\mathrm{mmHg}$ & $136 \pm 4$ & $133 \pm 5$ & $138 \pm 4$ & $137 \pm 5$ \\
\hline Diastolic blood pressure & $\mathrm{mmHg}$ & $81 \pm 3$ & $79 \pm 4$ & $82 \pm 4$ & $81 \pm 4$ \\
\hline \multicolumn{6}{|l|}{ Comorbidity } \\
\hline CVD & $\mathrm{n}(\%)$ & $8(40)$ & - & $9(45)$ & - \\
\hline Hyperlipidemia & $\mathrm{n}(\%)$ & $5(25)$ & - & $6(30)$ & - \\
\hline Hypertension & $\mathrm{n}(\%)$ & $7(35)$ & - & $8(40)$ & - \\
\hline Diabetes mellitus & $\mathrm{n}(\%)$ & $14(70)$ & - & $13(65)$ & - \\
\hline \multicolumn{6}{|l|}{ Medicines } \\
\hline Anti-thrombosis & $\mathrm{n}(\%)$ & $8(40)$ & $8(40)$ & $9(45)$ & $9(45)$ \\
\hline Statins & $\mathrm{n}(\%)$ & $5(25)$ & $5(25)$ & $6(30)$ & $6(30)$ \\
\hline$\beta$-blockers & $\mathrm{n}(\%)$ & $6(30)$ & $6(30)$ & $8(40)$ & $8(40)$ \\
\hline $\mathrm{ACEI} / \mathrm{ARB}$ & $\mathrm{n}(\%)$ & $4(20)$ & $4(20)$ & $4(20)$ & $4(20)$ \\
\hline $\mathrm{Ca}^{2+} \mathrm{CB}$ & $\mathrm{n}(\%)$ & $5(25)$ & $5(25)$ & $4(20)$ & $4(20)$ \\
\hline Sulfonylurea & $\mathrm{n}(\%)$ & $13(65)$ & $13(65)$ & $12(60)$ & $12(60)$ \\
\hline DDP-4 inhibitor & $\mathrm{n}(\%)$ & $4(20)$ & $4(20)$ & $5(25)$ & $5(25)$ \\
\hline SGLT2 inhibitors & $\mathrm{n}(\%)$ & $2(10)$ & $2(10)$ & $3(15)$ & $3(15)$ \\
\hline \multicolumn{6}{|l|}{ Ankle-brachial index (ABI) } \\
\hline \multirow[t]{2}{*}{ Duplex ultrasonography } & $\mathrm{R}$ (ratio) & $0.85 \pm 0.02$ & $0.92 \pm 0.01 *+$ & $0.87 \pm 0.01$ & $0.88 \pm 0.01$ \\
\hline & $\mathrm{L}$ (ratio) & $0.86 \pm 0.01$ & $0.93 \pm 0.01 *^{+}$ & $0.85 \pm 0.01$ & $0.85 \pm 0.01$ \\
\hline \multirow[t]{2}{*}{ Sphygmometry } & $\mathrm{R}$ (ratio) & $0.86 \pm 0.01$ & $0.93 \pm 0.02 *^{+}$ & $0.86 \pm 0.02$ & $0.89 \pm 0.01$ \\
\hline & $\mathrm{L}$ (ratio) & $0.87 \pm 0.01$ & $0.94 \pm 0.02 *^{+}$ & $0.85 \pm 0.01$ & $0.86 \pm 0.02$ \\
\hline
\end{tabular}

Values are mean \pm SEM. CET; cycling exercise training; GR, general rehabilitation; Pre, pre-intervention; Post, post-intervention; BMI, body mass index; CVD, cardiovascular diseases; $\mathbf{C a}^{2+} \mathbf{C B}$, channel blockers; DPP-4, Inhibitors of dipeptidyl peptidase 4 inhibitors; ACEI/ARB, angiotensin converting enzyme inhibitor/angiotensin II receptor blocker; R. right; L, left. ${ }^{*} P<0$. 05, Pre vs. Post; $+P<0.05$, GR+CET vs. GR. 
Table 2: Effects of general rehabilitation with or without cycling exercise training on cardiopulmonary responses to exercise and health-related quality of life in patients with peripheral arterial disease

\begin{tabular}{|c|c|c|c|c|c|}
\hline & & \multicolumn{2}{|c|}{ GR+CET } & \multicolumn{2}{|c|}{ GR } \\
\hline & & Pre & Post & Pre & Post \\
\hline \multicolumn{6}{|c|}{ Peak exercise performance } \\
\hline Work-rate & watt & $52.3 \pm 2.5$ & $76.4 \pm 2.5^{*^{+}}$ & $52.4 \pm 1.9$ & $57.7 \pm 2.1$ \\
\hline Hear rate & bpm & $114 \pm 2$ & $128 \pm 2^{*+}$ & $113 \pm 2$ & $114 \pm 2$ \\
\hline Stroke volume & $\mathrm{mL}$ & $64.1 \pm 1.8$ & $77.9 \pm 3.2^{*^{+}}$ & $66.2 \pm 3.1$ & $72.4 \pm 3.8$ \\
\hline Cardiac output & $\mathrm{L} / \mathrm{min}$ & $7.3 \pm 0.2$ & $9.8 \pm 0.3^{*^{+}}$ & $7.4 \pm 0.3$ & $7.8 \pm 0.3$ \\
\hline $\mathrm{V}_{\mathrm{E}}$ & $\mathrm{L} / \mathrm{min}$ & $33.9 \pm 1.6$ & $49.4 \pm 1.6^{*^{+}}$ & $33.8 \pm 1.2$ & $37.3 \pm 1.7$ \\
\hline $\mathrm{VO}_{2}$ & $\mathrm{~mL} / \mathrm{min}$ & $12.3 \pm 0.6$ & $18.0 \pm 0.6^{*^{+}}$ & $12.4 \pm 0.4$ & $13.6 \pm 0.6$ \\
\hline $\mathrm{VCO}_{2}$ & $\mathrm{~mL} / \mathrm{min}$ & $14.5 \pm 0.7$ & $20.1 \pm 0.9^{*^{+}}$ & $14.3 \pm 0.5$ & $15.6 \pm 0.7$ \\
\hline $\mathrm{V}_{\mathrm{E}}-\mathrm{VCO}_{2}$ slope & unit & $38.8 \pm 1.2$ & $34.2 \pm 0.8^{*++}$ & $38.1 \pm 1.9$ & $38.3 \pm 1.7$ \\
\hline MET & score & $3.52 \pm 0.17$ & $5.14 \pm 0.17^{*^{+}}$ & $3.52 \pm 0.13$ & $3.88 \pm 0.17$ \\
\hline \multicolumn{6}{|l|}{ 6-minute walk test } \\
\hline Initial pain & meter & $217.6 \pm 8.5$ & $323.5 \pm 19.8^{*^{+}}$ & $219.0 \pm 11.6$ & $211.2 \pm 13.2$ \\
\hline Terminal & meter & $363.0 \pm 17.7$ & $451.0 \pm 11.0^{*^{+}}$ & $370.8 \pm 18.5$ & $346.8 \pm 16.4$ \\
\hline
\end{tabular}

Short form-36 health survey questionnaire

$\begin{array}{llllll}\text { Physical } & \text { score } & 37.1 \pm 2.0 & 43.1 \pm 2.2^{*^{+}} & 38.4 \pm 2.2 & 39.5 \pm 2.3 \\ \text { Mental } & \text { score } & 35.2 \pm 2.2 & 42.8 \pm 2.1^{*^{+}} & 35.2 \pm 2.5 & 36.3 \pm 2.5\end{array}$

Values are mean \pm SEM. CET; cycling exercise training; GR, general rehabilitation; Pre, preintervention; Post, post-intervention; MET, metabolic equivalent of task, $\mathbf{V O}_{2}, \mathrm{O}_{2}$ consumption; $\mathbf{V E}$, minute ventilation; $\mathbf{V C O} 2, \mathrm{CO}_{2}$ production; ${ }^{*} P<0.05$, Pre vs. Post; $+P<0$. 05, GR+CET vs. GR. 
Table 3: Effects of general rehabilitation with or without cycling exercise training on blood cell counts in patients with peripheral arterial disease

\begin{tabular}{cccccc}
\hline & & \multicolumn{2}{c}{ GR+CET } & \multicolumn{2}{c}{ GR } \\
\cline { 3 - 6 } & & Pre & Post & Pre & Post \\
\hline Hematocrit & $\%$ & $40.6 \pm 2.2$ & $42.6 \pm 1.2$ & $41.60 \pm 1.7$ & $40.5 \pm 1.4$ \\
Erythrocyte & $10^{6} / \mu \mathrm{l}$ & $4.52 \pm 0.20$ & $4.79 \pm 0.15$ & $4.56 \pm 0.19$ & $4.52 \pm 0.17$ \\
Hemoglobin & $\mathrm{g} / \mathrm{dL}$ & $13.5 \pm 0.5$ & $13.95 \pm 0.4$ & $13.6 \pm 0.6$ & $13.3 \pm 0.4$ \\
Leukocyte & $10^{3} / \mu \mathrm{l}$ & $7.78 \pm 0.48$ & $6.30 \pm 0.29^{*}$ & $7.40 \pm 0.44$ & $6.87 \pm 0.37$ \\
Neutrophil & $10^{3} / \mu \mathrm{l}$ & $5.30 \pm 0.40$ & $3.78 \pm 0.21^{*}$ & $5.09 \pm 0.32$ & $4.87 \pm 0.27$ \\
Lymphocyte & $10^{3} / \mu \mathrm{l}$ & $1.75 \pm 0.14$ & $1.70 \pm 0.11$ & $1.62 \pm 0.11$ & $1.51 \pm 0.11$ \\
Monocyte & $10^{3} / \mu \mathrm{l}$ & $0.40 \pm 0.03$ & $0.41 \pm 0.02$ & $0.44 \pm 0.03$ & $0.40 \pm 0.02$ \\
Eosinophil & $10^{3} / \mu \mathrm{l}$ & $0.29 \pm 0.07$ & $0.19 \pm 0.03$ & $0.26 \pm 0.08$ & $0.26 \pm 0.05$ \\
Basophil & $10^{3} / \mu \mathrm{l}$ & $0.042 \pm 0.003$ & $0.034 \pm 0.002$ & $0.036 \pm 0.003$ & $0.040 \pm 0.004$ \\
Platelet & $10^{3} / \mu \mathrm{l}$ & $240 \pm 21$ & $234 \pm 13$ & $225 \pm 19$ & $242 \pm 20$ \\
Neutrophil/Lymphocyte & $\mathrm{ratio}$ & $3.42 \pm 0.37$ & $2.22 \pm 0.13^{*+}$ & $3.24 \pm 0.16$ & $3.25 \pm 0.18$ \\
\hline
\end{tabular}

Values are mean \pm SEM. CET; cycling exercise training; GR, general rehabilitation; Pre, preintervention; Post, post-intervention; $* P<0.05$, Pre vs. Post; $+P<0.05$, GR+CET vs. GR. 